\title{
Cataract Eye Prediction using Machine Learning
}

\author{
Shruthi Bhat \\ Dept of Computer Engg \\ PCCOE, Pune
}

\author{
Som Mosalagi \\ Dept of Computer Engg \\ PCCOE, Pune
}

\author{
Tejal Bhalerao \\ Dept of Computer Engg \\ PCCOE, Pune
}

\author{
Pushpak Katkar \\ Dept of Computer Engg \\ PCCOE, Pune
}

\author{
Rahul Pitale \\ Dept of Computer Engg \\ PCCOE, Pune
}

\begin{abstract}
Humans, being visually oriented, witness the happenings in the surroundings with the help of eyes. In the current scenario, blindness, and visual impairment has become a major and ubiquitous health problem. Although new technologies are rapidly progressing, visual impairment remains a noteworthy problem for worldwide healthcare systems. One of such problems is Cataract. Cataracts causing poor vision may also result in an increased risk of falling and depression. Earlier, it was usual among old age people, but now childhood cataract has become an important cause of blindness and severe visual impairment in children. Existing studies have been done mostly on Fundus image datasets for automatic detection of cataract and grading using a predefined feature set. The challenge is to detect cataract using the normal lens images at an early stage thus allowing people to test for cataract themselves. This would rather ensure that people belonging to remote areas need not reach out to ophthalmologists, just to check whether the person is facing a cataract problem or not. This paper uses CNN models taking normal lens image input for detection of cataract problems.
\end{abstract}

\section{Keywords}

Cataract detection, CNN, Fundus, Keras, Non-cataract, Normal lens image, TensorFlow.

\section{INTRODUCTION}

Humans, being visually familiarized, witness the happenings within the surroundings with the assistance of eyes. Within the current state of affairs, sightedness and handicap became major and omnipresent health issues. Though new technologies square measure apace progressing, handicap remains a motivating downside for worldwide attention systems. One among such issues is Cataract. A cataract could be a development of clouding up of the attention lens that ends up in a decrease in vision. Cataracts typically develop bit by bit that will have an effect on one or each eye. Symptoms could embrace pale colors, indistinct or visual disorder, halos around light-weight, bother with bright lights, and bother seeing in the dark. This might end in bother, Whereas, reading, driving, or recognizing faces. Poor vision because of cataracts may additionally end in Associate in Nursing hyperbolic risk of falling and depression. Cataracts cause half of all cases of sightedness, and thirty-third of handicap worldwide. Cataracts square sometimes measure because of ageing however may additionally occur because of radiation exposure, or trauma, or is also gift from birth, or occur following eye surgery for different issues. Risk factors of cataract embrace smoking, diabetes, tobacco prolonged exposure to daylight and alcohol. Earlier, it was usual among adulthood folks. However, currently childhood cataract has become one among the foremost vital causes of sightedness and severe handicap in youngsters. Thus, detection of cataract is the primary demand, and it will be done using exploitation varied strategies.

The branch of machine learning called Deep learning is a virtual representation of working of the human brain. Deep learning methods have created an impact of giving results better than human experts.Deep learning methods like Convolutional Neural Networks, Deep Neural Networks are used in various fields like medical imaging, search engine, gmail, computer vision, NLP, machine translation and many more. Deep learning models mostly depend on convolutional neural networks. Google's TensorFlow library can be used to build deep learning models with the help of dataflow graphs. This allows us to create many layers in a neural network. In order to build our Tensorflow model, Keras library can be used. Keras is simple that consists of an optimized interface for common use cases. Performance is an important measure to be considered in machine learning. Using high level APIs and fast debugging, TensorFlow gives good performance. Keras is the high level API used with TensorFlow to build the model. This can be used for classification, prediction, understanding, creation and discovery.[1]

\section{RELATED WORK}

Method of classification of cataract mainly comprises four parts namely preprocessing, feature extraction, feature selection and classifier or model.[5] The primary purpose of any classifier is achieved based on the input provided to it. Input for Cataract classification is a dataset of eye images of two different classes called cataract and non cataract. For many years, research on Fundus image analysis has been conducted. Fundus images are obtained by Fundus camera which clearly distinguishes cataract from normal eye.[2] The five convolution layer based on deep learning is used to separate the features in Fundus images.[4] Some of the features of retinal Fundus images have been extracted such as texture, sketch, color, wavelet, acoustical, spectral parameters and so on.[2] One of the other methods for cataract classification and detection is the Squeeze Net model. The dataset collected is first preprocessed in order to remove the noise in the images. To detect the circle part in the image, the Hough Circle algorithm is used. The training model is built using a convolutional neural network. Transfer Learning is used where a model developed for a task is reused as the beginning for another model.[3] In few other models, image preprocessing is done with the help of maximum entropy method. The features are extracted automatically using caffe. The extracted features must be identified and compared. In 
this case, Softmax and support vector machines are used for classification. For the collected dataset, four different classifications are done. But Softmax gives better accuracy[4].

A Convolutional neural network works well for object recognition. This system uses a pre-trained model with the image set for diabetic retinopathy. The trained models that are already available provide class objects which take an image as input.[1] Pool 5 layer feature mapping is used to classify and extract features. This feature extraction is cross-checked by using another method giving two conclusions. The first one provides the G-filter method that is used to overcome the reflection and interference of uneven illumination. The second one is used to increase the number of images available to improve accuracy.[6] Cataract must be detected as soon as possible so that it will be easy to prevent it by getting more and turning the person blind. The aim of this paper is to efficiently use a deep convolutional neural network to detect and classify cataracts. Existing automatic methods for cataract grading, such as [7-12], usually utilize a predefined set of image features that provides a redundant, or maybe noisy, incomplete representation. Moreover, all the predefined features were artificially extracted, a heuristic (need professional knowledge) method that depends on experience and luck. Also, its regulation takes a lot of time.

\section{SYSTEM ARCHITECTURE}

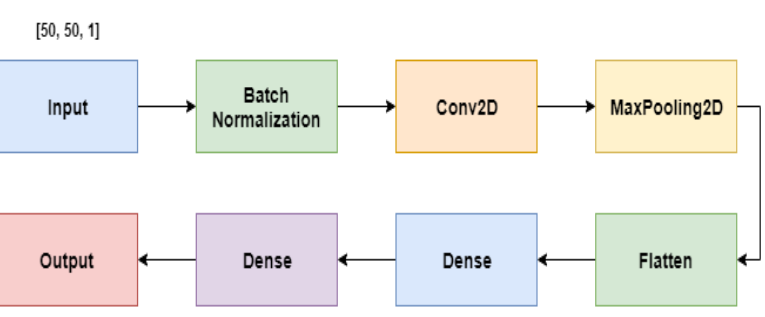

[1]

Fig 1: CNN model

The model takes an eye image dataset as input that is processed by subsequent layers of convolutional neural networks. Batch Normalization technique trains neural networks that standardize the input images to a layer batch wise thus stabilizing the process of learning. This decreases the number of training epochs that are required to train networks. 2D Convolutional Layer creates a convolution kernel that coevolves with the layer input and produces a tensor of outputs. Max Pooling down-samples an input image to reduce its dimensions. It allows making assumptions about features contained in the binned sub-regions. The featured map obtained is pooled and then flattened. The pooled feature map matrix is transformed into a single column by flattening which is further fed to the neural network for processing. Dense layer is responsible for adding the fully connected layer to the neural network.

The implementation methodology begins from a collection of good image dataset for both the classes namely cataract and normal eye. Image preprocessing is done involving 2 stepsresizing the images to a specific size and cropping the image borders. Due to a very high fluctuation in the size of images, all images need to be downsized to a common size of width 50 pixels and height 50 pixels. A model or a binary classifier is created in order to make predictions. This model is trained by feeding a training dataset to the model. The model is supposed to predict whether the image belongs to class cataract or non-cataract. The images of the data set used for testing is not the same as that used for training as the model may memorize the unimportant features of the training images. This issue is known as over fitting, and to avoid it we keep a portion of our dataset out of the training procedure. After the testing phase, the model is ready to predict for new images.(fig.2.)

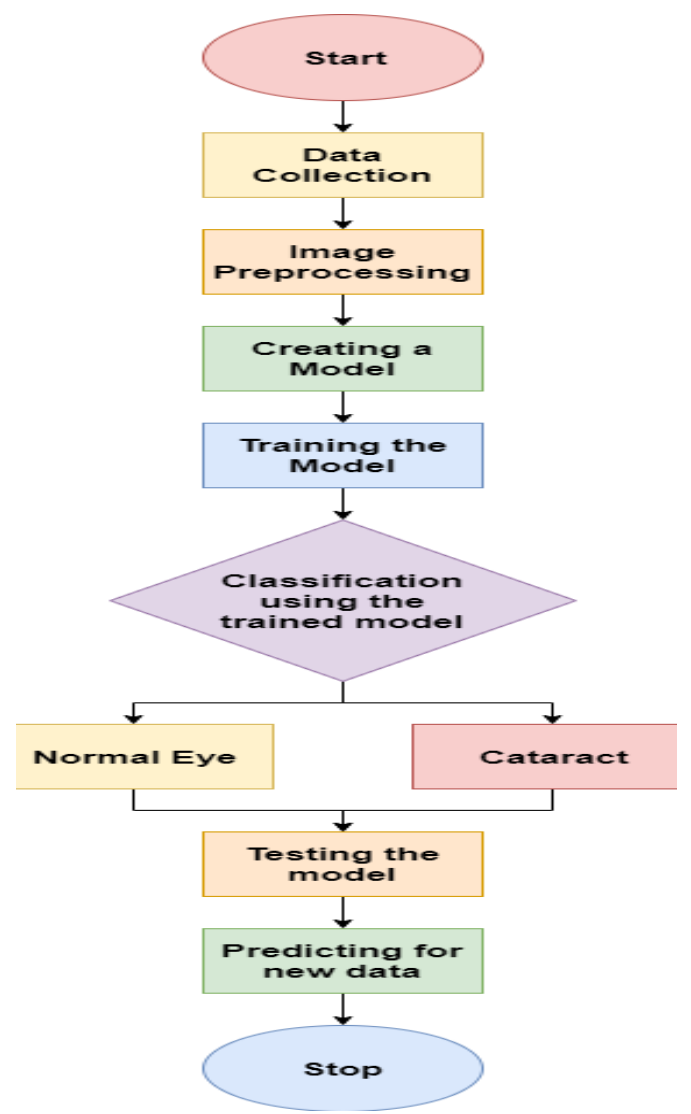

Fig 2: Implementation methodology

\section{PROPOSED SOLUTION}

The proposed solution takes input image that crops the eye part in order to obtain a valid image for classification. This image is available in binary format. Using flask middleware, image is converted into hexadecimal format and fed to the ML model. The model predicts the result in text format and it is sent to the user as output.

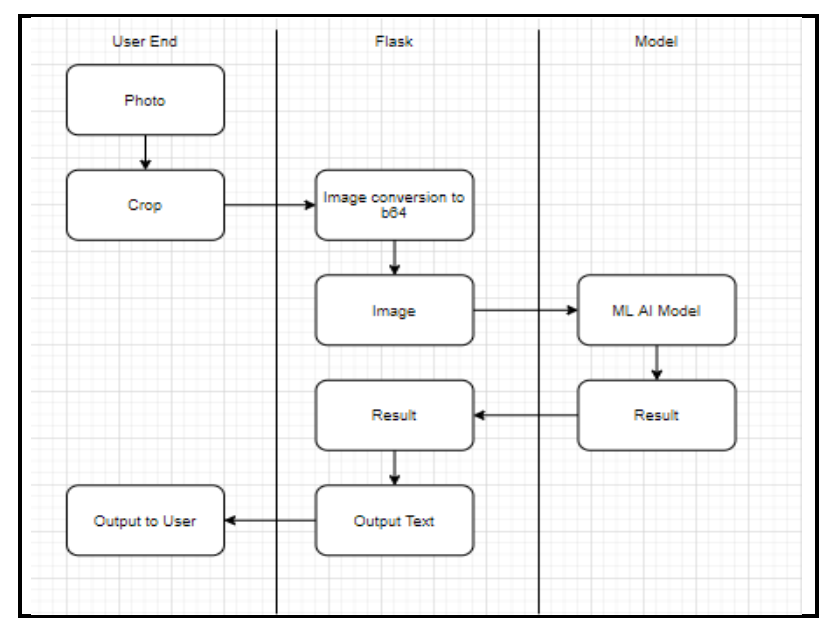

Fig 3: Proposed Solution 


\section{CONCLUSION}

In this paper, we proposed Convolutional neural network method to classify images into two classes namely cataract and non-cataract. The dataset has been collected and classified as cataract and non-cataract eye. Out of all the images, some are kept for training and some for testing. The model is trained with the training dataset and tested with the testing dataset to identify and classify the cataract in the new input image successfully.

\section{REFERENCES}

[1] Gowrishankar Sahana, Identification and Classification of Cataract Stages in maturity individuals victimization Deep Learning formula 2770, International Journal of Innovative Technology and Exploring Engineering (IJITEE), Volume-8 Issue-10, August 2019.

[2] Linglin Zhang et al, Automatic cataract detection and grading victimization Deep Convolutional Neural Network, IEEE ordinal International Conference on Networking, Sensing and management (ICNSC), Calabria, 2017.

[3] Evan, Xingzhi, Qian, Justin and Tingying Helen Zeng, Machine Learning on Cataracts Classification victimization Squeeze Net, fourth International Conference On Universal Village, Boston, USA, October 2018.

[4] Qinyan Zhang, Zhiqiang Qiao, Yanyan Dong and JiJiang rule, Classification of Cataract structure pictures supported Deep Learning, IEEE International Conference on Imaging Systems and Techniques, Beijing, China, pp $1-5$, October 2017

[5] J. V. B. Soares, J. J. G. Leandro, and R. M. Cesar, Retinal vessel segmentation victimization the 2-D physicist ripple and supervised classification. Medical Imaging, IEEE Transactions on Medical Imaging, vol.
25.

[6] Jianqiang Lia, He Hana, Linglin, i Zhangb, Bo Liua, Ch'ing Wangc and Jijiang Yangc, Automatic Cataract Detection and Grading victimization Deep Convolutional Neural Network, Ordinal International Conference on Networking, Sensing and management, Calabria, Italy, May 2017.

[7] J. J. Yang, J. Q. Li, R. F. Shen, Y. Zeng, J. He, J. Bi, et al., Exploiting ensemble learning for automatic cataract detection and grading, laptop ways and programs in biomedicine, vol. 124, 2016.

[8] H. Q. Li, J. H. Lim, J. Liu, P. Mitchell, A. G. Tan, J. J. Wang, et al., A computer-aided designation system of nuclear cataract, IEEE Transactions on medical specialty Engineering, vol. 57, 2010.

[9] M. Caixinha, E. Velte, M. Santos, and J. B. Santos, New approach for objective cataract classification supported ultrasound techniques victimization Multiclass SVM classifiers, IEEE International Ultrasonic conference, 2014.

[10] J. Zheng, L. Y. Guo, L. H. Peng, J. Q. Li, J. J. Yang, and Q. F. Liang, Fundus image primarily based cataract classification, 2014 IEEE International Conference on Imaging Systems,2014.

[11] W. M. Fan, R. F. Shen, Q. Y. Zhang, J. J. Yang, and J. Q. $\mathrm{Li}$, Principal part Analysis primarily based Cataract Grading and Classification, IEEE seventeenth International Conference on E-Health Networking, Applications and Services, pp. 459-462, 2015.

[12] L. Y. Guo, J. J. Yang, L. H. Peng, J. Q. Li, and Q. F. Liang, A computer-aided health care system for cataract classification and grading supported structure image analysis, Computers in business, vol. 69,2015. 